\title{
NONEXISTENCE OF COREFLECTIONS AND COPRODUCTS IN SOME SUBCATEGORIES OF SEMIGROUPS
}

\author{
P. G. TROTTER
}

\begin{abstract}
It is shown here that there are no nontrivial free regular semigroups, free regular semigroups on a semigroup or free products of regular semigroups. Analogous results are obtained in the categories of simple, bisimple and bisimple regular semigroups.
\end{abstract}

1. Introduction. Let $C$ denote a subcategory of the category of semigroups. Denote by $C(X)$ a coreflection object in $C$ for the set $X$, by $C(S)$ a coreflection object in $C$ for a semigroup $S$ and by $C(\chi)$ a coproduct object for the family $\chi=\left\{S_{i} ; i \in I\right\}$ in $C$ [4]. In accordance with usual terminology these are respectively a free $C$-semigroup, a free $C$-semigroup on a semigroup and a free product in C. Call $C(X), C(S)$ or $C(\chi)$ trivial if $X=\square, S$ is an object of $C$ or $|I| \leqslant 1$ respectively.

There are subcategories $C$ of semigroups in which these objects exist for any sets, semigroups and families in $C$. In particular they exist in the categories of semigroups and inverse semigroups [1], [6], [3], [5]. It is proved in this paper, for $C$ the category of regular semigroups, that there are no nontrivial objects $C(X), C(S)$ or $C(\chi)$. Analogous results for the categories of simple, bisimple and bisimple regular semigroups are provable but since the proofs in these cases are essentially similar to that for regular semigroups, they are not presented in detail.

2. The construction. Let $S$ be a semigroup. In this section a regular semigroup $\bar{S}$ is constructed so that $\bar{S}$ has regular subsemigroups $R$ and $T$ with $R \cap T=S$. With this construction the claimed results can be proved. The construction is based on that described in [3].

Choose a set of distinct elements $Z_{1}=\left\{x_{a}, y_{a} ; a \in S\right\}$ so that $Z_{1} \cap S=\square$. Let $X_{1}=\left\{x_{a} ; a \in S\right\}$ and $Y_{1}=\left\{y_{a} ; a \in S\right\}$. We have $X_{1} \cap Y_{1}=\square$. Denote by $F\left(Z_{1}\right)$ the free semigroup on $Z_{1}$. Let $F_{1}=S * F\left(Z_{1}\right)$ be the free product of $S$ and $F\left(Z_{1}\right)$. Put $H_{1}=S * F\left(X_{1}\right)$ and $K_{1}=S * F\left(Y_{1}\right)$. Let $\tau_{1}$ be the symmetric relation on $F_{1}$ generated by $\left\{\left(a x_{a} a, a\right),\left(a y_{a} a, a\right) ; a \in S\right\}$ and let $\rho_{1}$ be the least congruence on $F_{1}$ that contains $\tau_{1}$. Write $S_{1}=F_{1} / \rho_{1}, R_{1}=H_{1} / \rho_{1}$ and $T_{1}=K_{1} / \rho_{1}$.

The aim initially is to prove that $R_{1} \cap T_{1}=(S) \rho_{1}^{\#} \simeq S$. Some definitions and results are required for this.

Note that $(u, v) \in \rho_{1}$ if and only if there exists $p_{i}, q_{i} \in F_{1}^{1}$ and $\left(u_{i}, v_{i}\right) \in \tau_{1}$ for $1 \leqslant i \leqslant n$ so that

$$
u=p_{1} u_{1} q_{1}, p_{1} v_{1} q_{1}=p_{2} u_{2} q_{2}, \ldots, p_{n} v_{n} q_{n}=v .
$$

Received by the editors February 16, 1978 and, in revised form, June 11, 1979.

AMS (MOS) subject classifications (1970). Primary 20M10. ( 1980 American Mathematical Society $0002-9939 / 80 / 0000-0401 / \$ 02.25$ 
If at step $i$ in this chain $u_{i}=a$ and $v_{i}=a z_{a} a$ we call the step and $z_{a}$ an insertion. If $u_{i}=a z_{a} a$ and $v_{i}=a$ call the step and $z_{a}$ a deletion. A term $z_{a} \in Z_{1}$ may appear several times in the free product expression for $u$. Index these occurrences and the occurrences of $z_{a}$ as insertions in the chain (1). If the indexed term $\left(z_{a}\right)_{m}$ appears as both an insertion and a deletion in (1), we say $z_{a}$ is an insertion-deletion in (1).

LEMMA 2.1. If $(u, v) \in \rho_{1}$ then there is a chain of form (1) for $u$, $v$ with no insertion-deletions.

Proof. Suppose there are insertion-deletions in (1). Choose $i, j$ so that $\left(z_{a}\right)_{m}$ is inserted at step $i$ and deleted at step $j$ and so that no insertion-deletions occur between steps $i$ and $j$. Call $\left(z_{a}\right)_{m}$ a central insertion-deletion. Then $u_{i}=a \in S, v_{i}=$ $a z_{a} a, u_{j}=a z_{a} a$ and $v_{j}=a$. From the definition of $F_{1}$ as a free product we have $\left(p_{i} a, p_{j} a\right),\left(a q_{i}, a q_{j}\right) \in \rho_{1}$. Suppose $p_{i} a$ is changed to $f$ at some step between $i$ and $j$. If $f$ is obtained by a deletion then $f \in F_{1}^{1} a$. Since there are no insertion-deletions between steps $i$ and $j$, if $f$ is obtained by an insertion then also $f \in F_{1}^{1} a$. Dual statements apply for changes in $a q_{i}$. Hence, for $i<h<j$ and some $f_{h}, g_{h} \in F_{1}^{1}$, it can be seen that either $p_{h}=f_{h} a\left(z_{a}\right)_{m} g_{h}$ where $g_{h} u_{h} q_{h}, g_{h} v_{h} q_{h} \in a F_{1}^{1}$ or $q_{h}=$ $f_{h}\left(z_{a}\right)_{m} a g_{h}$ where $p_{h} u_{h} f_{h}, p_{h} v_{h} f_{h} \in F_{1}^{1} a$. Define respectively either $\bar{p}_{h}=f_{h} g_{h}$ and $\bar{q}_{h}=q_{h}$ or $\bar{p}_{h}=p_{h}$ and $\bar{q}_{h}=f_{h} g_{h}$. Then

$$
\begin{aligned}
u & =p_{1} u_{1} q_{1}, \ldots, p_{i-1} v_{i-1} q_{i-1}=\bar{p}_{i+1} u_{i+1} \bar{q}_{i+1}, \ldots, \bar{p}_{j-1} v_{j-1} \bar{q}_{j-1} \\
& =p_{j+1} u_{j+1} q_{j+1}, \ldots, p_{n} v_{n} q_{n}=v
\end{aligned}
$$

is a chain of form (1) which has less insertion-deletions than (1). So the lemma can be proved by induction.

REMARK 2.2. Note that by this lemma it follows that $S \simeq(S) \rho_{1}^{\#}$.

Lemma 2.3. Suppose $u \in H_{1}, v \in K_{1}$ and $(u, v) \in \rho_{1}$. There is a chain of form (1) for $u, v$ with no insertion-deletions and so that deletions from $F\left(X_{1}\right)$ precede insertions from $F\left(Y_{1}\right)$.

Proof. Assume, by Lemma 2.1, that (1) has no insertion-deletions. Hence (1) involves only deletions from $F\left(X_{1}\right)$ and insertions from $F\left(Y_{1}\right)$. Suppose $u_{i}=b, v_{i}=$ $b\left(y_{b}\right)_{h} b, u_{i+1}=a\left(x_{a}\right)_{k} a$ and $v_{i+1}=a$ where $y_{b} \in F\left(Y_{1}\right)$ and $x_{a} \in F\left(X_{1}\right)$. Let $w=$ $p_{i} v_{i} q_{i}=p_{i+1} u_{i+1} q_{i+1}$. Assume, in the free product expression for $w$, that $\left(x_{a}\right)_{k}$ precedes $\left(y_{b}\right)_{h}$; a similar argument applies otherwise. Then $w=p_{i+1} a\left(x_{a}\right)_{k} e\left(y_{b}\right)_{h} b q_{i}$ where $e=a f=g b$ and $p_{i}=p_{i+1} a\left(x_{a}\right)_{k} g, q_{i+1}=f\left(y_{b}\right)_{h} b q_{i}$, for some $e, f, g \in F_{1}^{1}$. We get

$$
p_{i} b q_{i}=p_{i+1} a\left(x_{a}\right)_{k} a f q_{i}, \quad p_{i+1} a f q_{i}=p_{i+1} g b q_{i}, \quad p_{i+1} g b\left(y_{b}\right)_{h} b q_{i}=p_{i+1} a q_{i+1} .
$$

Defining $\bar{p}_{i}=p_{i+1}, \bar{q}_{i}=f q_{i}, \bar{p}_{i+1}=p_{i+1} g, \bar{q}_{i+1}=q_{i}, \bar{u}_{i}=u_{i+1}, \bar{v}_{i}=v_{i+1}, \bar{u}_{i+1}=u_{i}$ and $\bar{v}_{i+1}=v_{i}$ then

$$
\begin{gathered}
u=p_{1} u_{1} q_{1}, \ldots, p_{i-1} v_{i-1} q_{i-1}=\bar{p}_{i} \bar{u}_{i} \bar{q}_{i}, \\
\bar{p}_{i} \bar{v}_{i} \bar{q}_{i}=\bar{p}_{i+1} \bar{u}_{i+1} \bar{q}_{i+1}, \quad \bar{p}_{i+1} \bar{v}_{i+1} \bar{q}_{i+1}=p_{i+2} u_{i+2} q_{i+2}, \ldots, p_{n} v_{n} q_{n}=v,
\end{gathered}
$$

which is a chain of form (1) with less inclusions from $F\left(Y_{1}\right)$ preceding deletions from $F\left(X_{1}\right)$ than (1) has. Hence the lemma can be proved by induction. 
REMARK 2.4. By Lemma 2.3, if $u \in H_{1}, v \in K_{1}$ and $(u, v) \in \rho_{1}$ then there exists $s \in S$ so that $(u, s) \in \rho_{1}$. Hence $R_{1} \cap T_{1}=(S) \rho_{1}^{\#}$.

Note that $S_{1}, R_{1}$ and $T_{1}$ are not regular semigroups. The desired semigroups $\bar{S}, R$ and $T$ will be constructed inductively to be regular "closures" of $S_{1}, R_{1}$ and $T_{1}$ respectively.

For the sake of the next step of the construction define an endomorphism $\alpha_{1}$ : $F_{1} \rightarrow F_{1}$ by $a \alpha_{1}=a$ and $y_{a} \alpha_{1}=x_{a}=x_{a} \alpha_{1}$ for each $a \in S, x_{a} \in X_{1}$ and $y_{a} \in Y_{1}$. Note that if $(u, v) \in \rho_{1}$ then the image under $\alpha_{1}$ of a chain of form (1) for $u$ and $v$ is a chain of form (1) for $(u) \alpha_{1}$ and $(v) \alpha_{1}$. Hence by the induced homomorphism theorem [1] there is a homomorphism $\beta_{1}: S_{1} \rightarrow R_{1}$ so that $\rho_{1}^{\#} \beta_{1}=\alpha_{1} \rho_{1}^{\#}$. Since $\alpha_{1}$ is the identity map on $H_{1}$ then $\beta_{1}$ is the identity map on $R_{1}$.

The construction can now be continued. Define a set of distinct elments $Z_{2}=\left\{z_{a} ; a \in S_{1}\right\}$ so that $Z_{2} \cap S_{1}=\square$. Let $X_{2}=\left\{z_{a} \in Z_{2} ; a \in R_{1}\right\}$ and $Y_{2}=$ $\left\{z_{a} \in Z_{2} ; a \in T_{1}\right\}$. Define $F_{2}=S_{1} * F\left(Z_{2}\right), H_{2}=R_{1} * F\left(X_{2}\right)$ and $K_{2}=$ $T_{1} * F\left(Y_{2}\right)$. Let $\tau_{2}$ be the symmetric relation on $F_{2}$ generated by $\left\{\left(a z_{a} a, a\right) ; a \in\right.$ $\left.S_{1}\right\}$ and let $\rho_{2}$ be the least congruence on $F_{2}$ containing $\tau_{2}$. Put $S_{2}=F_{2} / \rho_{2}, R_{2}=$ $H_{2} / \rho_{2}$ and $T_{2}=K_{2} / \rho_{2}$.

It will now be shown that $R_{2} \cap T_{2}=(S) \rho_{1}^{\#} \rho_{2}^{\#} \simeq S$.

Lemmas 2.1 and 2.3 can be proved as above if in their statements $\rho_{2}, H_{2}, K_{2}, X_{2}$ and $Y_{2}$ replace $\rho_{1}, H_{1}, K_{1}, X_{1}$ and $Y_{1}$ respectively. So $\left(S_{1}\right) \rho_{2}^{\#} \simeq S_{1}$ and in particular, by Remark $2.2,(S) \rho_{1}^{\#} \rho_{2}^{\#} \simeq S$.

Suppose $u \in H_{2}, v \in K_{2}$ and $(u, v) \in \rho_{2}$. By the generalisation of Lemma 2.3 and Remark 2.4, there exists $s \in S_{1}$ so that $(u, s) \in \rho_{2}$, and there is a chain of form (1) for $u$ and $s$ involving only deletions from $F\left(X_{2}\right)$. Let $\alpha_{2}: F_{2} \rightarrow F_{2}$ be the endomorphism defined by $a \alpha_{2}=b, z_{a} \alpha_{2}=z_{b}$ for any $z_{a} \in F_{2}, a \in S_{1}$ and $b=$ $a \beta_{1}$. Then $\alpha_{2}$ is the identity map on $H_{2}$. By considering the image under $\alpha_{2}$ of the chain (1) for $u$ and $s$ we see that $u=u \alpha_{2} \rho_{2} s \alpha_{2} \in R_{1}$. Likewise $v \rho_{2}^{\#} \in\left(T_{1}\right) \rho_{2}^{\#}$. Since $\left(S_{1}\right) \rho_{2}^{\#} \simeq S_{1}$ then $R_{2} \cap T_{2}=\left(R_{1} \cap T_{1}\right) \rho_{2}^{\#} \simeq S$.

Let $\beta_{2}: S_{2} \rightarrow R_{2}$ be the induced homomorphism so that $\rho_{2}^{\#} \beta_{2}=\alpha_{2} \rho_{2}^{\#}$.

Since $\rho_{1}^{\#}$ and $\rho_{2}^{\#}$ restricted to $S$ and $S_{1}$ respectively are injective homomorphisms, assume for convenience that $S \subseteq S_{1} \subseteq S_{2}$. We may similarly construct $S_{3}, R_{3}$ and $T_{3}$ so that $S_{2} \subseteq S_{3}, R_{3} \cap T_{3}=S$ and each element of $S_{2}, R_{2}$ and $T_{2}$ has an inverse in $S_{3}, R_{3}$ and $T_{3}$ respectively. Continuing by induction, semigroups $S_{n}, R_{n}$ and $T_{n}$ can be constructed so that $S_{n-1} \subseteq S_{n}, R_{n} \cap T_{n}=S$ and each element of $S_{n-1}, R_{n-1}$ and $T_{n-1}$ has an inverse in $S_{n}, R_{n}$ and $T_{n}$ respectively.

To complete the construction let $\bar{S}=\cup_{i=1}^{\infty} S_{i}, R=\cup_{i=1}^{\infty} R_{i}$ and $T=\cup_{i=1}^{\infty} T_{i}$. These semigroups are regular, $R \subseteq \bar{S} \supseteq T$ and $R \cap T=S$.

THEOREM 2.5. If $S$ is a nonregular semigroup then there exists no free regular semigroup on $S$.

Proof. Suppose $F$ is a free regular semigroup on $S$. Then there is a homomorphism $\alpha: S \rightarrow F$ so that for any homomorphism $\beta: S \rightarrow V, V$ a regular semigroup, there is a unique homomorphism $\gamma: F \rightarrow V$ where $\alpha \gamma=\beta$. By the construction $S$ is embeddable in a regular semigroup $\bar{S}$ with regular subsemigroups $R$ and $T$ so that 
$R \cap T \simeq S$. Let $V=\bar{S}$ and $(S) \beta=R \cap T$. Since $(F) \gamma$ is regular, while $S$ is not, there are at least two distinct choices for the map $\gamma$; namely with $(F) \gamma \subseteq R$ or $(F) \gamma \subseteq T$.

COROLLARY 2.6. There are no nontrivial free regular semigroups.

Proof. If there is a free regular semigroup on a set $X$ then it can be readily seen that there is a free regular semigroup on the free semigroup $F(X) . F(X)$ is nonregular.

COROLlARY 2.7. There are no nontrivial free products in the category of regular semigroups.

Proof. Let $F$ be a free product in the category of semigroups of the family $\left\{S_{i} ; i \in I\right\}$ of regular semigroups. It can be easily checked that a free product of this family in the category of regular semigroups exists if and only if the free regular semigroup on $F$ exists.

3. Some analogous cases. If $C$ is the category of simple, bisimple or bisimple regular semigroups the construction of $\$ 2$ can be remodelled to give analogous results. These categories are defined by existential conditions, as is the category of regular semigroups. For example, a semigroup $B$ is bisimple if and only if for each $a, b \in B$ there exists $x_{a b}, y_{a b}, u_{a b}, v_{a b} \in B$ so that $x_{a b} b u_{a b}=a, y_{a b} a v_{a b}=b$ and $b u_{a b}=y_{a b} a$.

For simple semigroups the construction can be readily modified. However, since the bisimple case is more complicated, a brief outline is presented here.

Let $S$ be a semigroup and $X_{1}$ be a set of distinct elements $X_{1}=$ $\left\{x_{a b}, y_{a b}, u_{a b}, v_{a b} ;(a, b) \in S \times S\right\}$ where $X_{1} \cap S=\square$. Define $Y_{1}$ similary so that $X_{1} \cap Y_{1}=\square$ and let $Z_{1}=X_{1} \cup Y_{1}$. Define $F_{1}, H_{1}$ and $K_{1}$ as in $\S 2$. Let $\tau_{1}$ be the symmetric relation on $F_{1}$ generated by the subset $\left\{\left(x_{a b} b u_{a b}, a\right),\left(y_{a b} a v_{a b}, b\right)\right.$, $\left.\left(b u_{a b}, y_{a b} a\right) ;(a, b) \in S \times S\right\}$ of $H_{1}$ and the corresponding subset of $K_{1}$. Let $\rho_{1}$ be the least congruence on $F_{1}$ containing $\tau_{1}$. In a chain of form (1) for $u, v \in F_{1}$, if $u_{i}=a$ and $v_{i}=x_{a b} b u_{a b}$ or $u_{i}=b$ and $v_{i}=y_{a b} a v_{a b}$ for $a, b \in S$ and $x_{a b}, y_{a b}, u_{a b}, v_{a b}$ $\in Z_{1}$, call step $i$ of (1) and $x_{a b}$ or $y_{a b}$ respectively an insertion. The reverse steps are deletions. Index the elements of $F\left(Z_{1}\right)$ in the chain (1) as in $\$ 2$. Suppose $\left(x_{a b}\right)_{m}$ is a central insertion-deletion; the transition involved in the insertion step being $a \rightarrow$ $\left(x_{a b}\right)_{m} b\left(u_{a b}\right)_{r}$. It can be checked that there is a chain of form (1), obtainable from (1), with $\left(x_{a b}\right)_{m}$ as a central insertion-deletion and with no step between the insertion and deletion of $\left(x_{a b}\right)_{m}$ that involves the transition $b\left(u_{a b}\right)_{r} \rightarrow y_{a b} a$. A similar statement applies for the other possible central insertion-deletions. With this observation, Lemma 2.1 can be readily generalised. The generalisation of the remainder of the construction is straightforward.

Note that the lack of symmetry in the existential conditions used to define bisimple semigroups is not essential. However with symmetrical conditions the proof of generalised Lemma 2.1 appears more cumbersome.

The construction for bisimple regular semigroups is a straightforward generalisation of the above. 
THEOREM 3.1. Let $C$ be the category of simple, bisimple or bisimple regular semigroups. Then there are no nontrivial $C(X), C(S)$ or $C(\chi)$ where $X$ is a set, $S$ is a semigroup and $\chi$ is a family of objects in $C$.

It is known [2] that free products of any families exist in the category $C$ of orthodox semigroups. It can therefore be readily checked that there exist free orthodox semigroups on some semigroups; for suppose $\chi$ is a family of orthodox semigroups and $G$ is a free product of $\chi$ in the category of semigroups, then $C(G)=C(\chi)$. However free orthodox semigroups do not exist on any nonempty set [7].

\section{REFERENCES}

1. A. H. Clifford and G. B. Preston, The algebraic theory of semigroups, Vols. 1 and 2, Math. Surveys, no. 7, Amer. Math. Soc., Providence, R. I., 1961, 1967. MR 36 \# 1558.

2. I. Imaoka, Free products of orthodox semigroups, Mem. Fac. Lit. Sci. Shimane Univ. Natur. Sci. 10 (1976), 1-6.

3. D. B. McAlister, A homomorphism theorem for semigroups, J. London Math. Soc. 43 (1968), 355-366. MR 37 \#329.

4. B. Mitchell, Theory of categories, Academic Press, New York, 1965. MR 34 \#2647.

5. G. B. Preston, Inverse semigroups: some open questions, Proceedings of a Symposium on Inverse Semigroups and their Generalisations (Northern Illinois Univ., DeKalb, Ill., 1973), Northern Illinois Univ., DeKalb, Ill., 1973, pp. 122-139. MR 54 \#2848.

6. H. E. Scheiblich, Free inverse semigroups, Proc. Amer. Math. Soc. 38 (1973), 1-7. MR 46 \#196.

7. P. G. Trotter, Free objects in some categories of semigroups, Conf. on the Algebraic Theory of Semigroups in Honour of A. H. Clifford (Tulane University, New Orleans, La., 1978).

Department of Mathematics, University of Tasmania, Hobart, Tasmania, Australia 